\title{
Chronische Rückenschmerzen
}

\section{Frühzeitig behandeln!}

_ „Ziel der Schmerztherapie ist es, eine rasche und anhaltende Schmerzlinderung bis hin zur Schmerzfreiheit zu erreichen", so Dr. Bettina Kern, Wiesbaden. Nur so könnten schmerzbedingte Einschränkungen vermieden und eine Schmerzchronifizierung verhindert werden. Die gelte insbesondere für Patienten mit chronischen Rückenschmerzen. Oft werde zu lange mit nebenwirkungsbelasteten nicht-steroidalen Antirheumatika experimentiert, bevor ein stark wirksames Opioid wie Tapentadol (Palexia $\left.{ }^{\varpi}\right)$ zum Einsatz komme. Dieses sollte so früh wie möglich, so lange wie nötig und ausreichend hoch dosiert eingesetzt werden. So könne die Chronifizierung verhindert werden. „Besser befristet knackig als lebenslang unzureichend behan-

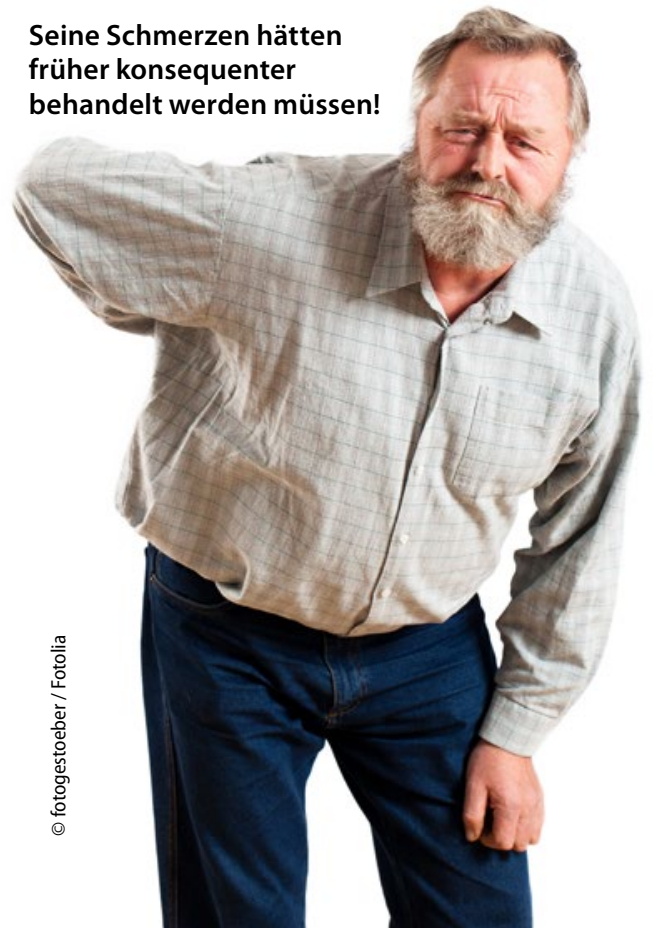

deln“, so Kern. Das Schmerzgedächtnis dürfe erst gar nicht zum Nachdenken kommen.

\section{Dualen Wirkmechanismus nutzen}

Die besondere Wirksamkeit von Tapentadol beruht auf seinem dualen Wirkmechanismus. Die Substanz ist zum einen ein $\mu$-Rezeptor-Agonist, wodurch die Überaktivität von Schmerzsignalen im peripheren und zentralen Schmerzsystem herunterreguliert wird. Zum anderen hemmt Tapentadol die Wiederaufnahme von Noradrenalin und stellt so die ausgefallene körpereigene Schmerzhemmung wieder her. „Unter Tapentadol kommt es bei Rückenschmerzen mit und ohne neuropathische Komponente $\mathrm{zu}$ einer schnellen und effektiven Schmerzreduktion bis hin zur Schmerzfreiheit“, so Dr. Lilit Flöther, Oberärztin an der anästhesiologischen Universitätsklinik in Halle/Saale. Die Substanz sei auch besser verträglich als andere gängige starke Schmerzmittel und lasse sich schnell und unproblematisch hoch- und herunterdosieren. Sie sei für junge und alte Menschen gleichermaßen geeignet.

Vorteilhaft für den verordnenden Arzt ist auch, dass für Tapentadol Rabattverträge mit den Krankenkassen ausgehandelt wurden, sodass die Verordnung dieser Substanz dem Wirtschaftlichkeitsgebot entspricht. Mit anderen Worten: Das individuelle Regressrisiko wird gesenkt.

Dr. Peter Stiefelhagen

- Pressegespräch „Chronischer Rückenschmerz und starke Analgetika in Praxis und Klinik: Standortbestimmung für Tapentadol"; Köln, Januar 2017 (Veranstalter: Grünenthal)

\section{Erstattungsbetrag für Umeclidinium vereinbart}

GlaxoSmithKline und der GKV-Spitzenverband haben sich auf einen Erstattungsbetrag für Incruse ${ }^{\circledast}$ (Wirkstoff: Umeclidinium) geeinigt. Das Präparat ist für die bronchialerweiternde Erhaltungstherapie zur Symptomlinderung bei erwachsenen Patienten mit COPD zugelassen. Der neue Erstattungsbetrag von 46,62 Euro (für die 30er-Packung) gilt seit 01. Februars 2017. Damit ist Incruse ${ }^{\circledR}$ derzeit das günstigste LAMA-Monotherapeutikum für COPD-Patienten.

Red.

- Nach Informationen von GSK

\section{Weniger Hypoglykämien durch $300 \mathrm{E} / \mathrm{ml}$ Insulin glargin}

Ziele der Insulingabe bei Diabetes mellitus sind eine optimale zirkadiane Basalabdeckung, eine physiologische Nüchternblutzucker-Kontrolle und eine geringe intraindividuelle Variabilität. Dies gelingt mit Insulin glargin $300 \mathrm{E} / \mathrm{ml}$ (Toujeo ${ }^{\circledast}$ ) gut. Das Insulin bildet nach der subkutanen Injektion ein kompaktes Depot mit einer reduzierten Oberfläche, weshalb die Insulinfreisetzung langsamer erfolgt und länger anhält als bei Insulin glargin $100 \mathrm{E} / \mathrm{ml}$. Aus diesem Grund sinkt auch das Risko für Hypoglykämien. -

Red

- Nach Informationen von Sanof

\section{Antivirales Kombi-Nasenspray}

Neue In-vitro-Daten sprechen dafür, dass das abschwellend wirkende und schleimhautpflegende Nasenspray mit Xylometazolin und Dexpanthenol (nasic ${ }^{\oplus}$ ) antivirale Effekte haben könnte. In einer In-vitro-Studie wurde eine Virusreduktion um vier LogStufen nachgewiesen. Das entspricht der Wirkung von Desinfektionsmtteln.

Red. 